\title{
A Feature-Based Semantic Model for Automatic Product Cost Estimation
}

\author{
Y.-S. Ma, N. Sajadfar, and L. Campos Triana
}

\begin{abstract}
To address the requirement of dynamic pricing and cost control in high-variation product manufacturing, nowadays many companies face the problem of generating quotes and order prices timely, accurately and consistently. A generic semantic model for the purpose of automatic cost estimation is proposed, in which a new concept named cost feature, is suggested. A cost feature can be identified with data mining methods for different targeted clients or products, and conceptually interfaced with product design and manufacturing features. Feature-based mapping model is used to determine feature scope and cost level defined, including all the dependency relations with other domain features. This model is expected to enable a visual, flexible and semantically consistent scheme to address effective and efficient product cost structures, frequent configuration variations and business changes. A case study is used to illustrate this new method. The preliminary study shows that the proposed method is potentially effective for manufacturers.
\end{abstract}

Index Terms - Cost estimation, semantic modeling, product pricing, associative feature, cost feature.

\section{INTRODUCTION}

In today's competitive world and the on-line business era, effective and timely cost estimation has a critical role in manufactory's success. In terms of practical applications, industry needs to quickly estimate the price for any new product configuration in a customer order either interactively or automatically by on-line applications. High cost estimation accuracy at the very early stage and effective cost control during the operation phase are essential for the smooth business operation. Quick and accurate cost estimation brings lots of advantages over competitors. Cost estimation can also affect all aspects of production processes and management decisions, such as choosing between producing the product components in-house or outsourcing the product.

There have been a number of researchers who studied on accurate cost estimation for manufacturing products which in turn is a part of cost engineering. Cost engineering also defines the process and conditions for forecasting a product's price [1]. There are also lots of methods and tools for doing

Manuscript received April 10, 2013; revised July 10, 2013. This work was supported in part by Canada NSERC discovery grant No. 355454-09 "Interoperability in knowledge-driven collaborative engineering" and MITACS Inc. Accelerate Cluster program, i.e. "Methodology research on business process reengineering with a neutral information on model of ERPs".

Y.-S. Ma and N. Sajadfar are with the University of Alberta, Edmonton, AB, Canada (e-mail: yongsheng.ma@ualberta.ca; sajadfar@ualberta.ca)

L. Campos Triana is with McCoy Drilling \& Completions, Farr, Edmonton, AB, Canada (e-mail: LCampos@mccoyglobal.com ). cost estimation. In general, cost estimation methods have two main categories: traditional and advanced approaches. Traditional cost estimation approach includes bottom-up engineering, analogy, and parametric methods. This approach is fundamentally based on historical data and experience knowledge.

Advanced cost estimation approach includes expert judgment, feature-base evaluation, fuzzy logic, neural networks and data mining (DM) methods. This approach is more suitable for complex product and dynamic cost estimation [2]. Cost estimation can be carried out in each stage of a product life cycle, such as conceptualization, concept design, detailed design, prototyping and manufacture. Many researches recognize that up to $80 \%$ of the product cost is determined at the conceptual and design phases [3]. Therefore, accurate cost estimation at early stages and with different levels of defined details has an important role in manufacture success.

The goal of this paper is to investigate a new manufacturing cost calculation model coherently throughout the lifecycle of a product series, especially emphasizing at the conceptual design stage, which integrates three functional sub-models: feature-based costing, data mining, and semantic reasoning.

\section{Challenge}

Manufacture cost estimation is a cluster of challenging tasks done in three levels: conceptual, design and manufacture. Although cost estimation is clearly related to the profitability, the systematic pricing was not adopted in most companies due to the complex associativity among the technical and business considerations. A systematic semantic model is required in order to tackle the relations and constraints among the product elements, processes, resources and actors.

Many manufacturers used to do their cost estimation at the manufacturing process level which belongs to the traditional way, like engineering bottom-up method [4]. In the manufacturing process level, all the expenses can be quantified already as the state of art of practice. For example, Donald R. Woods mentioned more than 20 factors in his book, such as: cost of fabricated equipment, cost of material, cost of labor, cost of control, cost of land, cost of storage, legal fees [5], etc. Although this traditional approach is still being used, however its drawback is pretty clear, that is when the product gets complex, the accuracy of cost estimation will decrease significantly. Also there are lots of uncertain factors behind all the cost elements, such as the changes of costs related to the changes of materials, processes and equipment items. Such uncertain dynamic changes can increase the risk 
of broad range of cost estimation, such as the risk of misleading loss-indication in a potentially profitable deal [6].

Cost estimation is a dynamic procedure that must be done periodically during each stage of a product lifecycle; and it has been the trend to compete on price at product conceptual specification and the early design level. So instant cost estimation is demanded at earlier stage and with more accuracy. However due to the lack of accurate information in early stage of a product order, the cost estimation is currently estimated with experience and subjects to modifications at the manufacture level.

The purpose of this paper is introducing a new cost estimation semantic model that can systematically associate design features and manufacturing features with a mechanism, i.e. the proposed cost features. They can serve as the active information agents and work out cost elements intelligently in the product specification stage with the inference capability from the cost models of associated features at the design and manufacture levels.

\section{Methodology}

Feature technology has been successfully applied in engineering computer tools in the past decades because of its capability to resemble engineer's semantic patterns related to their design and manufacturing threads. Feature is a kind of data structure that can describe more information related to the all aspects of the product that was not described in traditional semantic attributes. Many advanced engineering design features and machining features have been successfully defined and applied. Feature can describe the material characteristics, machine methods, shape, etc.

Feature-base cost estimation was introduced in 1990's and a lot of research was done in this area. The feature-based cost estimation approach entails to identify cost related features that can be defined as associated to certain functions and manufacturing processes. For example, a part's cost can be linked to the design features for the estimation of material cost, power, optional add-ons, and other quantifiable impacts. Cost can also be linked to manufacture features to estimate the machine utilization and process time cost [7]. However, so far in practice, identifying, defining and the tracking the dependencies and impacts of changes related to design and machining features at each level of cost estimation are very complicated procedures and are almost impossible for complex product. The authors contemplate to define a new type of features that is Cost Feature, a feature object class, aiming to cluster, encapsulate, update and manage those cost-related dependencies, rules, constraints and referenced features defined from other domains, such as those related to design functions and machining processes. The Cost Feature Association method is introduced in this work to map the cost features with other types of features in cost estimation via a set of associative and semantic trees.

For identifying cost feature, different types of information is required, such as the historical data, uncertainly factors, design and manufacture conditions and etc. Knowledge-based techniques like Data Mining (DM) can recognize all the product information from historical data, such as their attributes and the correlations and relationship patterns among their attributes. The next phase is cost feature establishment with attribute selection that is one of the most important steps. Given the fact that not all the information of the product is useful for cost estimation, most effective attributes have to be selected for this purpose [8]. Such selected attributes are then associated by using a decision tree. In the next step, the rules for data analyses and weight of importance are set. Finally, DM can present the data pattern that can predict the future information from historical data. These algorithms are built into the methods of cost feature class and derivatives of their object instances. Also, other supporting functions of the cost feature are developed to enable the intelligent automation procedure and to persistently store information patterns as well as the attributes that was discovered by data mining, such as manufactory specification and inventory information.

To explore the scenarios of applying cost features, semantic modeling is useful. Semantic modeling is a new knowledge representation approach that expresses real world objects in the form of predefined terms and entities that are interpretable by modern computer program such that the real world entities and their dynamic behavior can be consistently described, modified and persistently reused within the prescribed scope of application. Semantic modeling is an extension of high level object-oriented and modular software development method that is useful for consistent software engineering modeling. It is one kind of knowledge modeling that describes the meaning of data from the point view of the computer entities with interactions and user interfaces. The objectives of semantic modeling are abstracting the knowledge and understanding the meaning of data and information. The other aim of semantic modeling is implementing the high cohesive model [9].

Semantic modeling is useful for mapping the entities from real to virtual world; modeling behaviors from complex life cycles of business, describing the dynamics of activities and illustrating the processes so that well-structured software elements, e.g. foundation classes, intermediate modules and functioning applications are modeled and implemented by object-oriented programming. Fig. 1 shows the overall concept semantic modeling for cost estimation.

Everyone can define his own semantic modeling, and everyone can have a different description of system. However, a disciplined semantic modeling approach for system implementation needs to follow several rules:

1) All the steps of semantic modeling have to be defined clearly.

2) The abstract model should create relations for mapping the information between the real and the virtual worlds.

3) The modeling objects have to be generically defined and directly mapped into class diagram.

4) A semantic model has to specify the relationships and interactions among the modeling objects to describe the logics.

Three kinds of data are analyzed, classified, and related with some predefined relations. The first kind is referred to as tangible data that are explicit attributes related to cost objects describing properties such as physical, natural and economic data. 


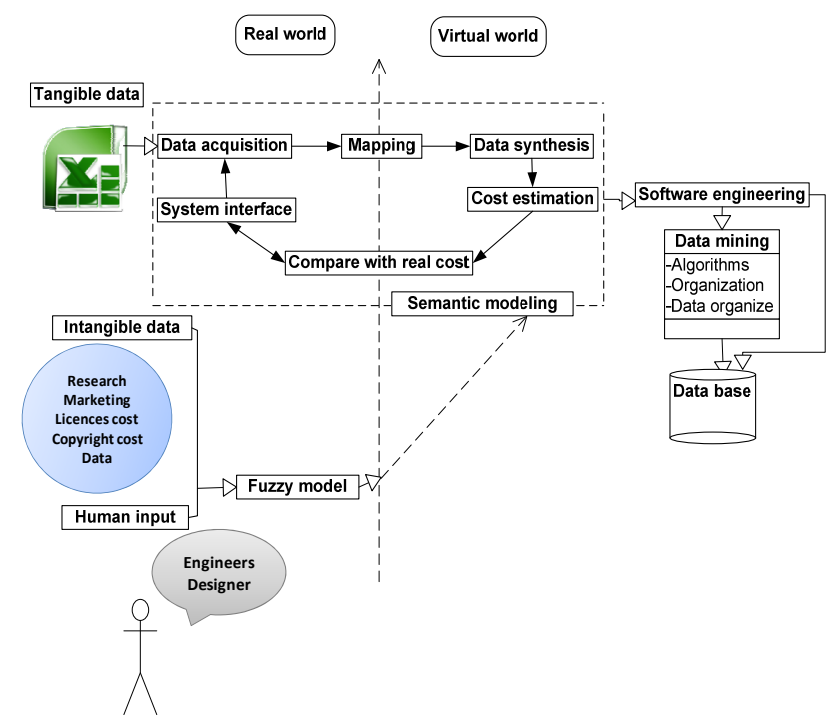

Fig. 1. Concept of semantic modeling for cost estimation

On the other hand, another kind, intangible data are those attributes that are not obviously or directly related to cost elements but implicitly associated that the relationships are to be discovered and defined dynamically like the data describing context, background, social status, organizational attributes and cultural data. The third kind of data is interactive and subjective that requires engineering inputs from marketing managers, designers, process engineers, and purchasers; this kind of data fills the gap between the tangible and intangible data types [10]. For doing reasonable and accurate cost estimation, all these three kinds of data are needed. Tangible data can be materialized in manufacturing view with $\mathrm{CAD} / \mathrm{CAM}$ data models, historical data, ERP data, geometry data, manufacturing capacity, assembly and completion data. Intangible data includes brand value, goodwill, training, skill requirement, marketing positioning, software licenses, IP copyrights, research and development cost, etc.

In the next step, the conceptual semantic relations illustrating the dependencies between cost features and other engineering information entities are to be defined and some elementary and non-exhaustive relations are shown in Fig. 2. Cost features, supported with a template library, is a conceptual class of objects that has to define the road map for doing the cost estimation within a specific scope. It has to define cost constituents, searching mechanisms and target elements in the scope and a sound cost structure. To interact with other types of features which are used for constraining the cost elements and rules, a unified feature scheme can be used. Such a unified feature system model covers a multi-facet feature-oriented platform which defines the basic referencing mechanisms and information inference scope which can include concept features, machining features, user-defined face-cluster features, design features or any other kind of features. Also, it has to define the level of details that is necessary for features and the structures that it wants to keep the feature dependency information. According to the scope and level of features, then the cost feature can be defined by identifying appropriate attributes and a set of constraints. Next, the tangible and intangible data sets are to be associated by identifying their various referencing sources, for instance, from $\mathrm{CAD} / \mathrm{CAM}$ data, product configurations, ERP data tables as well as references to licenses, product models, historical data and suppliers' data. From CAD/CAM model and product configuration, design features can be determined. A design feature includes all the information about the material requirement, functional assembly features, functional part features and part geometry constriction features. On the other hand manufacturing costs can be determined from machining features and machining processes. Machining features includes all the information about the machines that are to be used to produce the specific product, such as a waterjet machine, surface grinding machine, a welding machine, $\mathrm{CNC}$ turning or milling centers.

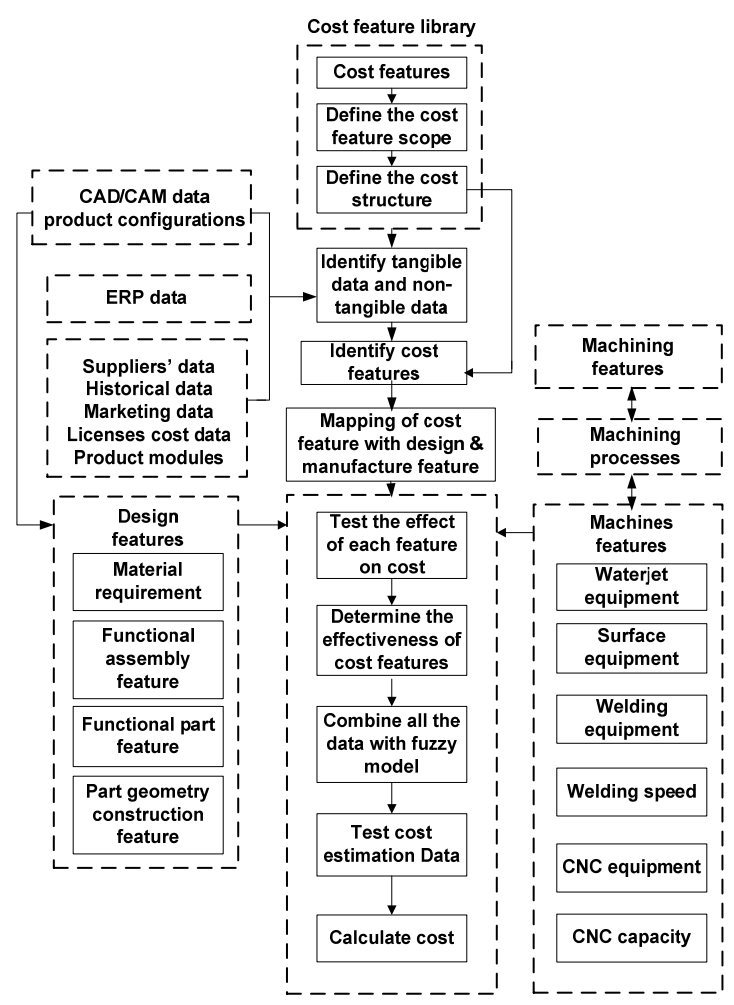

Fig. 2. Cost feature related elementary semantics with some non-exhaustive relations

Their specific data sets can be easily searched and used such as welding speed, equipment hourly rate and $\mathrm{CNC}$ programming capacity. The most important step following is the mapping of cost feature attributes with design and manufacture feature and testing their cost effect by using a unified weighting scheme. Then the variations of the effective cost features can be fully defined and the total product cost and the price quote will be determined. At the end, all the data need to be factored in with a unified fuzzy model to evaluate the sensitivities towards changes of context and conditions as well as the reasonability of cost estimation model for different cases.

\section{CASe Study}

A real industrial case of oil and gas equipment was selected for a case study. A Tong is commonly-used equipment for holds and provides the torque to the drilling pipes on an oil rig. 


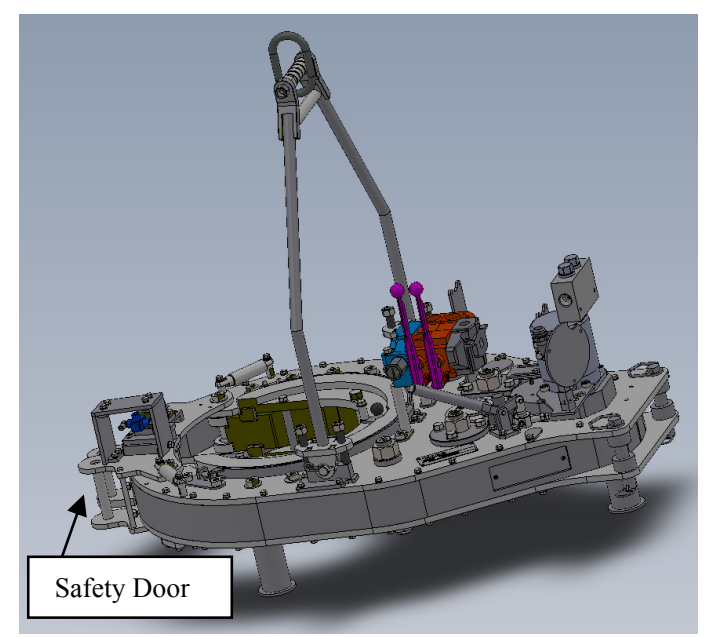

Fig. 3. Example Tong Assembly Body 3D CAD model

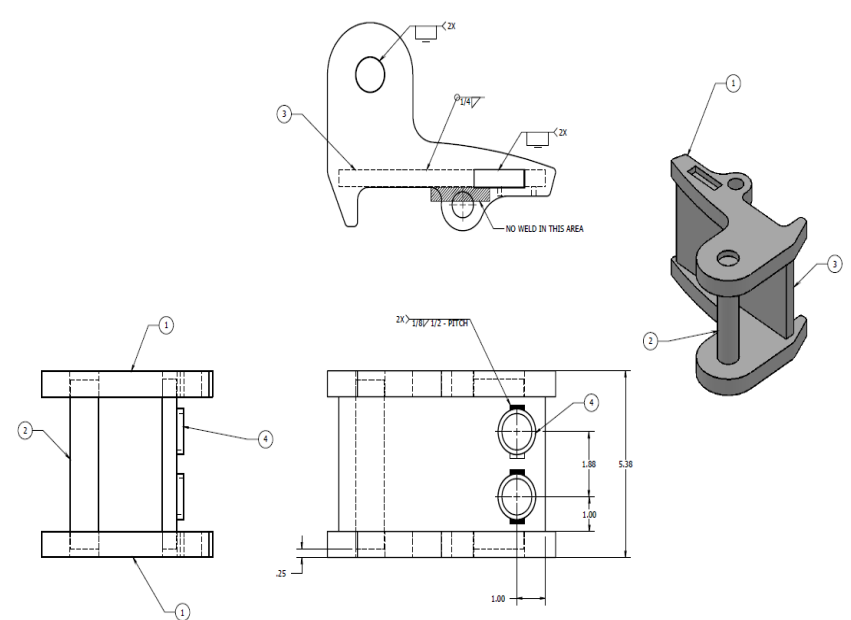

Fig. 4. Safety Door model

TABLE I: ERP INFORMATION ABOUT SAFETY DOOR PART

\begin{tabular}{ccccccc}
\hline Item & $\begin{array}{c}\mathrm{Q} \\
\mathbf{T}\end{array}$ & Title & $\begin{array}{c}\text { Material } \\
\text { Cost } \mathbf{( \$ )}\end{array}$ & $\begin{array}{c}\text { Labor } \\
\text { Cost } \\
\mathbf{( \$ )}\end{array}$ & $\begin{array}{c}\text { Burden } \\
\text { Cost } \\
(\$)\end{array}$ & $\begin{array}{c}\text { Time } \\
\text { Spend } \\
\text { (min) }\end{array}$ \\
\hline 1 & 2 & Latch & 24.17 & 15.7 & 5.6 & 30 \\
2 & 1 & Hinge & 8.7 & 12 & 1.14 & 20 \\
3 & 1 & Spacer & 27 & 8.4 & 2.17 & 12 \\
4 & 2 & Holder & 11 & 3.84 & 0.7 & 3 \\
\hline
\end{tabular}

Fig. 3 shows a Tong body assembly in a 3D CAD model. According to the industry's historical data, the safety door is a critical part of tong body. Because the safety door is an important part of the product that the end users will touch to open and close during drilling operation and to maintain the tong body. The safety door is not a complex part but is welded together from six plates. Fig. 4 shows the elementary plates of the safety door weldment, and Table I shows the cost of each part that was determine in manufacture level.

In the current costing system of the company investigated, the ERP information shows the material, labor and burden costs for each item. However, there is no information about feature cost from the ERP database. In this case, if the company wants to change any of the design features, there is no systematic method to estimate the cost with new feature.

For describing our new feature-based semantic cost modeling method, at first, the cost feature has to be recognized. Fig. 5 shows the parts and the face elements of the cost feature for the safety door. For example SD1 and SD2 in Part D can be produced by cutting away the extra notches from the big blank part or welding the small blocks on a smaller blank prepared; both options can be evaluated from the input data extracted from the machining feature properties and using the relevant cost feature functions defined in relationship to the critical face elements.

Fig. 6 shows that SA3 and SA2 have a critical relation with each other as a result the weight of this relation is higher than the other features. SA2, SA3 is a hinge of control for safety door. According to the interfacing feature relation schema, we can define the weight as an attribute for the relevant cost feature [11].

For example the weight of SA3 $\left(\mathrm{W}_{\mathrm{SA} 3}\right)$ is 4 , because it has 4 connections with the other feature. There for, any changes on SA3 has cost effect on 4 more features, however any changes on SB3 just has an effect on 1 feature.

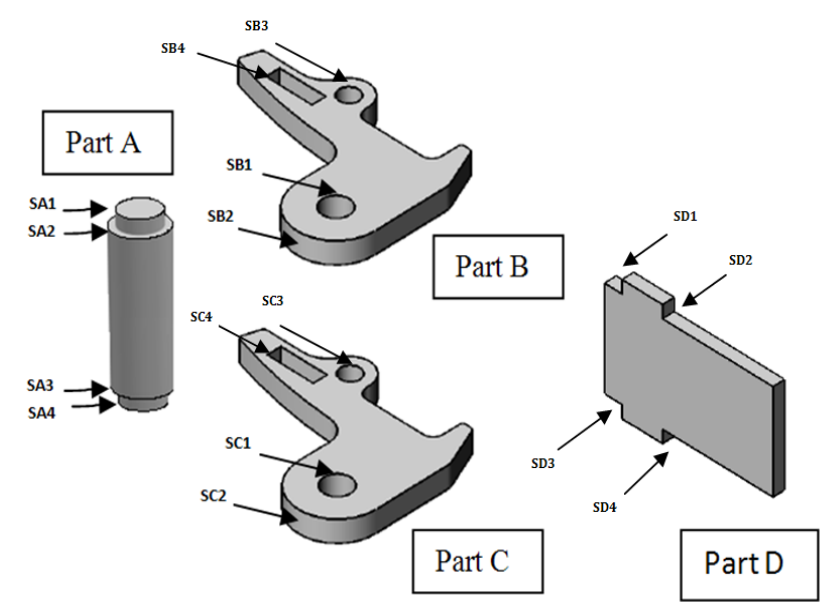

Fig. 5. Cost feature elements for the safety door module

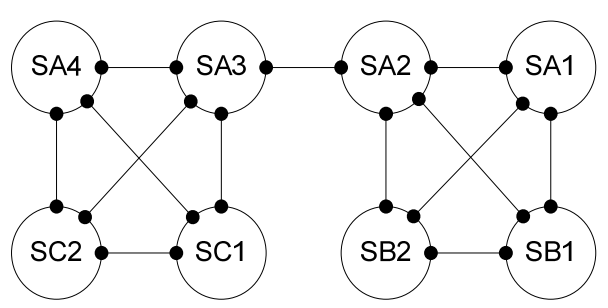

Fig. 6. Interfacing feature faces between parts A, B and C

TABLE II: COST FEATURE INFORMATION

\begin{tabular}{|c|c|c|c|c|c|c|c|}
\hline Part & Face & $\mathrm{W}_{\mathrm{i}}$ & $\begin{array}{c}\text { Thickness } \\
\text { (in) }\end{array}$ & $\begin{array}{c}\text { Min. feed rate } \\
\text { (in/hr) }\end{array}$ & Quality & $\begin{array}{c}\text { Max. feed } \\
\text { rate (in/hr) }\end{array}$ & Quality \\
\hline A & SA1 & 3 & $1.2 \sim 1.5$ & 50 & Good & 75 & Fair \\
\hline A & SA2 & 4 & $1.2 \sim 1.5$ & 30 & Good & 50 & Fair \\
\hline A & SA3 & 4 & $1.2 \sim 1.5$ & 30 & Good & 50 & Fair \\
\hline A & SA4 & 3 & $1.2 \sim 1.5$ & 50 & Good & 75 & Fair \\
\hline B & SB1 & 3 & $0.75 \sim 1$ & 50 & Good & 75 & Fair \\
\hline B & SB2 & 3 & $0.75 \sim 1$ & 30 & Good & 50 & Fair \\
\hline B & SB3 & 1 & $0.75 \sim 1$ & 30 & Good & 50 & Fair \\
\hline B & SB4 & 2 & $0.75 \sim 1$ & 10 & Good & 25 & Fair \\
\hline C & SC1 & 3 & $0.75 \sim 1$ & 50 & Good & 75 & Fair \\
\hline C & SC2 & 3 & $0.75 \sim 1$ & 30 & Good & 50 & Fair \\
\hline C & SC3 & 1 & $0.75 \sim 1$ & 30 & Good & 50 & Fair \\
\hline C & SC4 & 2 & $0.75 \sim 1$ & 10 & Good & 25 & Fair \\
\hline D & SD1 & 2 & $0.5 \sim 0.7$ & 30 & Good & 50 & Fair \\
\hline D & SD2 & 2 & $0.5 \sim 0.7$ & 30 & Good & 50 & Fair \\
\hline D & SD3 & 2 & $0.5 \sim 0.7$ & 30 & Good & 50 & Fair \\
\hline D & SD4 & 2 & $0.5 \sim 0.7$ & 30 & Good & 50 & Fair \\
\hline
\end{tabular}




\section{CONCLUSION}

In this paper, a new feature-based semantic model has been proposed for cost estimation purpose. This model is built on top of three sub models: feature-based association mapping, data mining and semantic modeling. Feature-based mapping model is used to determine feature scope and cost level defined, including all the dependency relations with other domain features such as conceptual, design, machining and user defined face cluster features. Data mining was proposed to identify correlations among tangible data, intangible data and human inputs for cost estimation purpose. Finally semantic modeling used to map the cost feature attributes with other predefined features, such as design and machining features. The future research will focus on mechanism implementation and full integration of the multi-facet feature models with the proposed automatic cost estimation model.

\section{ACKNOWLEDGMENT}

The authors would like gratefully acknowledge the technical case study material provided by McCoy Drilling and Completions, Farr. The research work reflected in this paper was mainly carried out at University of Alberta, Edmonton, Canada.

\section{REFERENCES}

[1] R. Roy, S. Kelvesjo, S. Forsberg, and C. Rush, "Quantities and Qualitative cost estimation for engineering design," Journal of Engineering Design, 2011

[2] C. G. Hart, Z. He, R. Sbragio, and N. Vlahopoulos, "An advanced cost estimation methodology for engineering systems," Systems Engineering, DOI: 10.1002/sys, 2011.

[3] A. R. Mileham, G. C. Currie, A. W. Miles, and D. T. Bradford, "A parametric approach to cost estimating at the conceptual stage of

design," Journal of Engineering Design, vol. 4, no. 2, pp. 117-125, 2007.

[4] J. W. Hermann, "Design for production: Concepts and applications," in Proceedings of the SME East Coast Region 3 Annual Member's Conference, 2003.

[5] D. R.Woods, Cost Estimation for the Process Industries, 1st ed. McMaster University, 1983.

[6] O. Trivailo, M. Sippel, and Y.A. Glu, "Review of hardware cost estimation methods, models and tools applied to early phases of space mission planning," Progress in Aerospace Science, 2012, doi:10.1016/j.paerosci.2012.02.001.

[7] A. Niazi, J. S. Dai, S. Balabni, and L. Seneviratne, "Product cost estimation: technique classification and methodology review", Journal of Manufacturing Science and Engineering, vol. 128, 2006.

[8] N. Al Khalidi, A. A. Saifan, and I. M. Alsmadi, "Selecting a standard set of attributes for cost estimation of software projects," in Proc. International Conference on Computer, Information and Telecommunication Systems (CITS), 2012.

[9] F. T. S. Chang, and J. Zhang, "Modeling for agile manufacturing systems," International Journal of Production Research, vol. 39, no. $11,2001$.

[10] G. L. H. Svendsen, and J. F. L. Sørensen, "There's more to the picture than meets the eye: measuring tangible and intangible capital in two marginal communities in rural Denmark," Journal of Rural Studies, vol. 23, pp. 453-471, 2007

[11] J. Han, M. Pratt, and W.C. Regli, "Manufacturing feature recognition from solid models: a status report," IEEE Transactions on Robotics and Automation, vol. 16, no. 6, 2000.

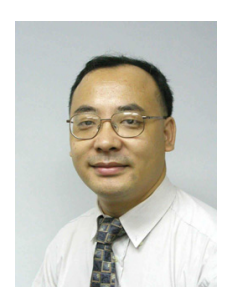

Singapore.

Dr. Ma serves as an editor of Advanced Engineering Informatics, Elsevier since 2012

Y.-S. Ma is a professor and a registered professional engineer with the Dept. of Mechanical Engineering, University of Alberta, Canada. His main research areas include feature-based product and process modeling, CADCAM, and product lifecycle management. He received his $\mathrm{Ph} . \mathrm{D}$. degrees from UMIST, UK in 1994. From 2000 to 2007, he had been an Associate Professor in the school of Mechanical and Aerospace Engineering, Nanyang Technological University, 